\title{
EFICACIA DE FUNGICIDAS PARA EL CONTROL DE MANCHAS FOLIARES EN SOJA EN EL DISTRITO DE MINGA GUAZÚ, PARAGUAY
}

\section{Efficacy of fungicides for late season soybean diseases in Minga guazú, Paraguay}

\author{
Enciso-Maldonado, Guillermo A. ${ }^{1}$; Maidana-Ojeda, Marco ${ }^{1}$; Machuca Aquino, Pablo R. ${ }^{2}$; Fernández- \\ Gamarra, Marta A. ; Schlickmann-Tank, José A. ${ }^{1 *}$ \\ ${ }^{1}$ Centro de Desarrollo e Innovación Tecnológica (CEDIT), Km 38 ruta 6 "Dr. Juan León Mallorquín”, \\ Hohenau, Departamento de Itapúa, CP 6290, Paraguay. ${ }^{2}$ Universidad Católica Nuestra Señora de la \\ Asunción Unidad Pedagógica Hohenau, Padre Guillermo Hütte esq. Nanawa, Obligado, Departamento \\ de Itapúa, CP 6290, Paraguay *E-mail: joseschlickmann2010@gmail.com
}

\begin{abstract}
RESUMEN
Las enfermedades son una de las mayores limitantes fitosanitarias en el cultivo de soja [Glycine max (L.) Merr.], entre ellas las enfermedades de final de ciclo (EFC) son las protagonistas en ausencia de la roya asiática de la soja (Phakopsora pachyrhizi). Este trabajo presenta los resultados de evaluaciones a campo de la eficacia de diferentes fungicidas en el control de las EFC en el cultivo de soja. El ensayo se realizó en Minga Guazú, Alto Paraná, Paraguay. Se utilizó la variedad NA 5909 RR. Los ingredientes activos evaluados fueron Bixafen + Protioconazole + Trifloxistrobin (T2), Picoxystrobin + Protioconazole (T3), Picoxystrobin + Cyproconazole (T4), Fluxapyroxad + Epoxiconazole + Pyraclostrobin (T5), (Benzovindiflupyr + Azoxistrobina) + Difenoconazole + Ciproconazole (T6), Mancozeb (T7) y Carbendazim (T8). Las aspersiones se realizaron según la ocurrencia de los estadios fenológicos R1, R3 y R5. Las principales variables evaluadas fueron la severidad, eficacia de control (EC) y rendimiento en $\mathrm{kg} \mathrm{ha}$ 1. La menor severidad se observó con el T5, mientras que T8 no fue efectivo para reducir la severidad; los tratamientos T5, T6 y T2 fueron los más eficaces para controlar las EFC, diferenciándose significativamente de los demás tratamientos. Los tratamientos T2, T3, T4, T5, T6 y T7 obtuvieron los mejores rendimientos y fueron estadísticamente similares. El T8 mostró el menor rendimiento y peso de mil semillas. En conclusión, la mayoría de los fungicidas presentaron un buen control sobre las EFC, así también sobre el rendimiento de la soja.
\end{abstract}

Palabras clave: Control químico, enfermedades de final de ciclo (EFC), carboxamidas, triazoles, estrobirulinas

\begin{abstract}
Diseases are one of the major phytosanitary constraints in soybean [Glycine max (L.) Merr.], among them, late-season disease complex is the most common one as a result of the absence of Asian soybean rust (Phakopsora pachyrhizi). This paper introduces the results of field evaluations of the efficiency of different fungicides to control late-season disease complex in soybean. The trial was carried out in Minga Guazú, Alto Paraná, Paraguay. The variety used was NA 5909 RR. The active ingredients evaluated were Bixafen + Prothioconazole + Trifloxystrobin (T2), Picoxystrobin + Prothioconazole (T3), Picoxystrobin + Cyproconazole (T4), Fluxapyroxad + Epoxiconazole + Pyraclostrobin (T5), (Benzovindiflupyr + Azoxystrobin) + Difenoconazole + Cyproconazole (T6), Mancozeb (T7) and Carbendazim (T8). Spraying was carried out according to the occurrence of phenological stages R1, R3, and R5. The main variables evaluated were severity, control efficiency (CE), and yield in $\mathrm{kg} \mathrm{ha}^{-1}$. The least severe late-season disease complex was observed with T5, while T8 was not effective in reducing severity; treatments T5, T6, and T2 were the most effective ones, differing significantly from the other treatments. Treatments T2, T3, T4, T5, T6, and T7 obtained the best yields and were statistically similar. T8 showed the lowest yield and 1000-grain weight. In conclusion, most fungicides showed efficient control on late-season disease complex, as well as on soybean yield.
\end{abstract}

Keywords: Chemical control, late-season disease complex, carboxamides, triazoles, strobilurins

Recibido: 5/Jul/2021. Aceptado: 1/Sept/2021 


\section{INTRODUCCIÓN}

Las enfermedades de final de ciclo (EFC) de la soja son causadas principalmente por un complejo de hongos patógenos que sobreviven en el aire, agua y suelo, saprófitos, cuya dispersión se realiza por medio de esporas (Hartman et al., 2015). Los principales daños ocasionados por las EFC son la reducción del área foliar fotosintéticamente activa, defoliación prematura, reducción de la eficiencia de intercepción de la radiación y de la tasa de crecimiento en el período crítico, madurez anticipada, disminución de la calidad de semillas, número y peso de granos y, por ende, del rendimiento (Zárate, 2017). Además, la prevalencia de estas enfermedades en el campo, dependen de las condiciones de manejo del cultivo y de las características climáticas de la zona (Ivancovich y Botta, 2003).

Entre las principales EFC se encuentran la mancha marrón (Septoria glycines), la mancha anillada (Corynespora casiicola), el tizón foliar y la mancha púrpura de la semilla (Cercospora spp.) y la mancha ojo de rana (Cercospora sojina) (Hartman et al., 2015). El manejo de estas enfermedades se realiza principalmente por medio de fungicidas. En Paraguay, la aplicación de fungicidas en el cultivo de soja está dirigida principalmente para el control de la roya asiática, utilizando fungicidas sitio-específicos (sistémicos) y multisitio (protectores) (Enciso-Maldonado et al., 2019). Sin embargo, la roya asiática no se manifiesta todos los años con la misma intensidad y en algunos años ni siquiera aparece en toda la temporada. Cuando ocurre tal situación, las enfermedades de final de ciclo (EFC) pasan a ser el principal objetivo de la aplicación de fungicidas. Las EFC han sido mayormente estudiadas en Argentina, Brasil y Uruguay, mientras que en Paraguay son escasos los reportes sobre eficacia de control de fungicidas. Por ello, se estableció como objetivo del trabajo evaluar la eficacia de control de diferentes fungicidas para el control de enfermedades de final de ciclo.

\section{MATERIALES Y MÉTODOS}

El trabajo se realizó en Minga Guazú, Departamento de Alto Paraná, Paraguay, en las coordenadas $54^{\circ}$ $82^{\prime} 27^{\prime \prime} \mathrm{S}, 54^{\circ} 82^{\prime} 37^{\prime \prime} \mathrm{O}$, entre octubre de 2020 y marzo de 2021.

Los tratamientos consistieron en la aplicación de diferentes pre-mezclas de fungicidas utilizados para el manejo de enfermedades de soja en Paraguay (Tabla 1). Se utilizó el diseño en bloques completos al azar con cuatro repeticiones. La unidad experimental se constituyó de 5 hileras de $5 \mathrm{~m}$ de largo distanciadas a $0,45 \mathrm{~m}$ (2,25 $\mathrm{m}$ de ancho), en donde se sembraron 15 semillas por metro lineal de la variedad NA 5909 RR. Se dejó 1 $\mathrm{m}$ de espacio entre unidades experimentales dentro de cada bloque y $3 \mathrm{~m}$ entre bloques, totalizando un área experimental total de $725 \mathrm{~m}^{2}$. En cada unidad experimental se delimitó $4.05 \mathrm{~m}^{2}$, eliminando $1 \mathrm{~m}$ de los extremos de cada unidad experimental y una hilera de cada borde.

Tabla 1. Tratamiento, fungicida y dosis utilizados en el ensayo.

\begin{tabular}{|c|c|c|}
\hline Tratamiento & Fungicida & Dosis \\
\hline $\mathrm{T} 1$ & Testigo (agua) & - \\
\hline $\mathrm{T} 2$ & Bixafen $12,5 \%$ + Protioconazole $17,5 \%$ + Trifloxistrobin 15 & $500 \mathrm{~mL} \mathrm{ha}^{-1}$ \\
\hline $\mathrm{T} 3$ & Picoxystrobin $10 \%+$ Protioconazole $11,67 \%$ & $600 \mathrm{~mL} \mathrm{ha}^{-1}$ \\
\hline $\mathrm{T} 4$ & Picoxystrobin $9 \%+$ Cyproconazole $4 \%$ & $600 \mathrm{~mL} \mathrm{ha}^{-1}$ \\
\hline $\mathrm{T} 5$ & Fluxapyroxad $5 \%+$ Epoxiconazole $5 \%+$ Pyraclostrobin 8,1 & $800 \mathrm{~mL} \mathrm{ha}^{-1}$ \\
\hline T6 & $\begin{array}{c}\text { (Benzovindiflupyr } 15 \%+\text { Azoxistrobina } 30 \% \text { ) + Difenocona- } \\
\text { zole } 25 \%+\text { Ciproconazole } 15 \%\end{array}$ & $200 \mathrm{~g} \mathrm{ha}^{-1}+250 \mathrm{~mL} \mathrm{ha}{ }^{-1}$ \\
\hline $\mathrm{T} 7$ & Mancozeb $80 \%$ & $1500 \mathrm{~g} \mathrm{ha}^{-1}$ \\
\hline $\mathrm{T} 8$ & Carbendazim $50 \mathrm{FW}$ & $400 \mathrm{~mL} \mathrm{ha}^{-1}$ \\
\hline
\end{tabular}

Los tratamientos fueron aplicados con una pulverizadora manual con erogación de $100 \mathrm{~L} \mathrm{ha}^{-1}$ a presión constante (30 bar), accionada mediante tubo de gas carbónico $\left(\mathrm{CO}_{2}\right)$ a una velocidad de $5 \mathrm{~km} \mathrm{~h}^{-1}$. Las aspersiones se realizaron según la ocurrencia de los estadios fenológicos R1, R3 y R5, los cuales se manifestaron a los $45(04 / 12 / 2020), 65(24 / 12 / 2020)$ y $83(11 / 01 / 2021)$ días después de la siembra.

Para monitorear enfermedades de final de ciclo se procedió a realizar un monitoreo periódico con una frecuencia de una semana en las plantas de las unidades experimentales. Cuando se detectaron síntomas de manchas foliares, se procedió a la observación a través del estereoscopio y microscopio para realizar un diagnóstico de la enfermedad. 
Se evaluó la severidad de manchas foliares, a los 7 días después de la última aplicación con ayuda de la escala diagramática de Martins et al. (2004), la cual cuenta con cinco niveles $(2,4 ; 5,2 ; 25 ; 40,5$ y 66,6 \%). A partir de los datos de severidad se calculó la eficacia de control (EC) de los tratamientos, utilizando la fórmula: $\mathrm{EC}=(($ Infección testigo - Infección tratamiento $) /$ Infección testigo $) * 100$. También se evaluó el porcentaje de defoliación con ayuda de la escala diagramática de defoliación de Hirano et al. (2010), la cual cuenta con seis niveles $(5,15,45,65,85$ y $100 \%)$. Por último, se evaluaron el rendimiento y el peso de mil semillas.

Los datos fueron evaluados sometidos al análisis de varianza y cuando se encontraron diferencias significativas entre los tratamientos, se realizó la prueba de comparación de medias de Tukey al $5 \%$.

\section{RESULTADOS}

\section{Condiciones ambientales}

$\mathrm{Al}$ inicio de la temporada de siembra se manifestó una sequía, lo cual pudo afectar la viabilidad y densidad de inóculo en el aire de enfermedades como la roya asiática de la soja, lo cual explicaría su ausencia en las plantas evaluadas. A partir de octubre, las lluvias fueron frecuentes y normales y la temperatura y humedad relativa fueron normales para el desarrollo de la soja (Toledo, 2018) (Figura 1).

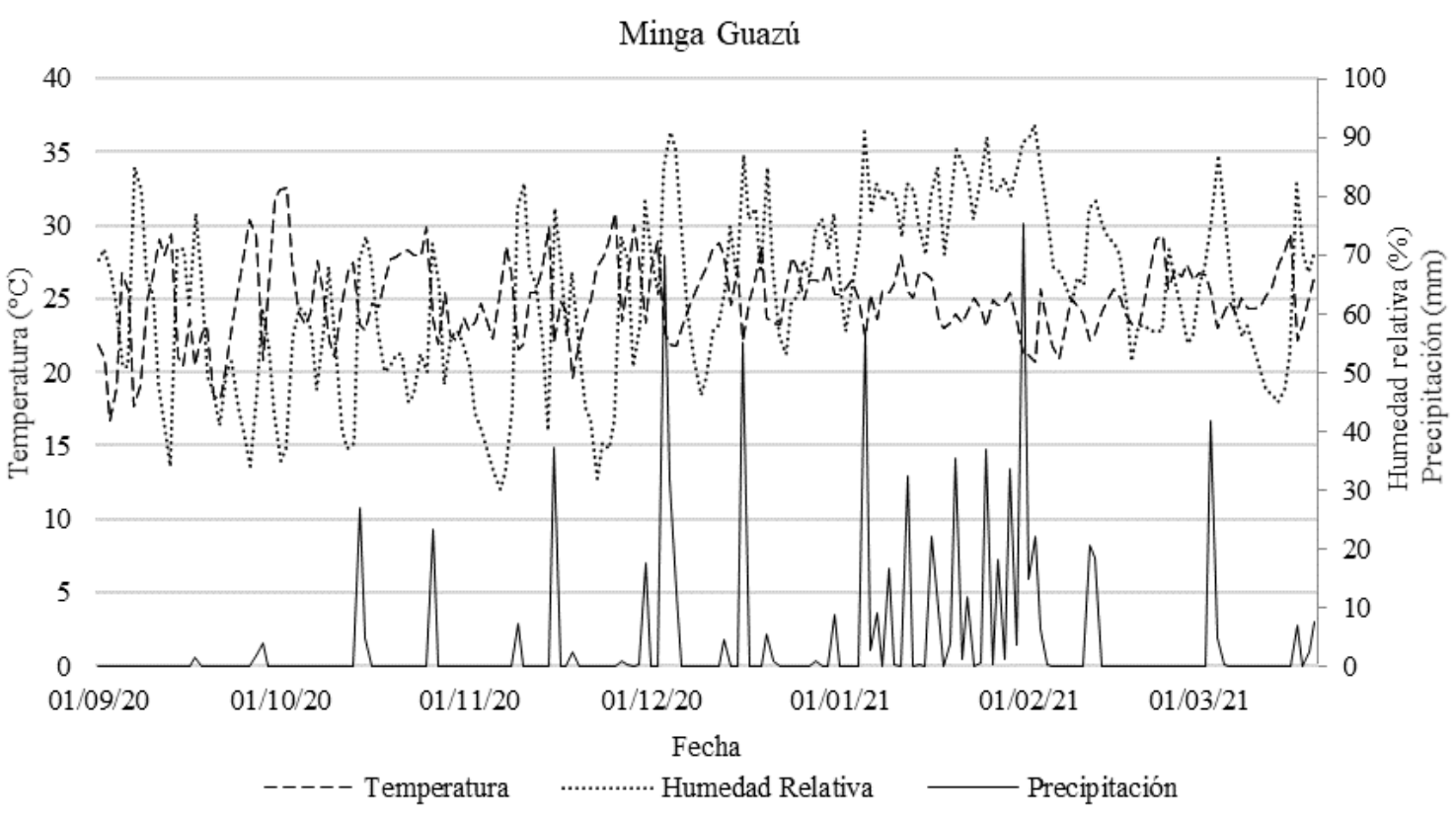

Figura 1. Temperatura, humedad relativa y precipitación en Minga Guazú, Paraguay. Temporada de cultivo de soja 2020/2021.

\section{Severidad de enfermedades de final de ciclo y eficacia de control de fungicidas}

La enfermedad de final de ciclo predominante durante el periodo experimental fue la mancha marrón causada por Septoria glycines. Sin embargo, se registraron síntomas de mildiu y mancha anillada, pero no sobrepasaron el $5 \%$ de severidad, por lo tanto, el complejo de enfermedades de final de ciclo se basó en las evaluaciones de mancha marrón. Se observó diferencias significativas entre los tratamientos para la severidad de las EFC, indicando que los tratamientos tienen un efecto sobre esta variable $(\mathrm{p}<0,0001)$. El porcentaje de severidad osciló entre 8,8 y $49,1 \%$. El mayor valor fue observado en el testigo que recibió agua en las aplicaciones, mientras que la menor severidad se observó con el tratamiento T5. Los fungicidas mantuvieron la severidad de la enfermedad entre 8,8 y $20,3 \%$. No se observaron diferencias significativas entre los tratamientos T2, T3, T4, T5, T6 y T7, mostrando el mismo efecto sobre esta variable, mientras que el tratamiento T8 fue menos efectivo para reducir la severidad, alcanzando un $20,3 \%$ de enfermedad (Figura 2). 


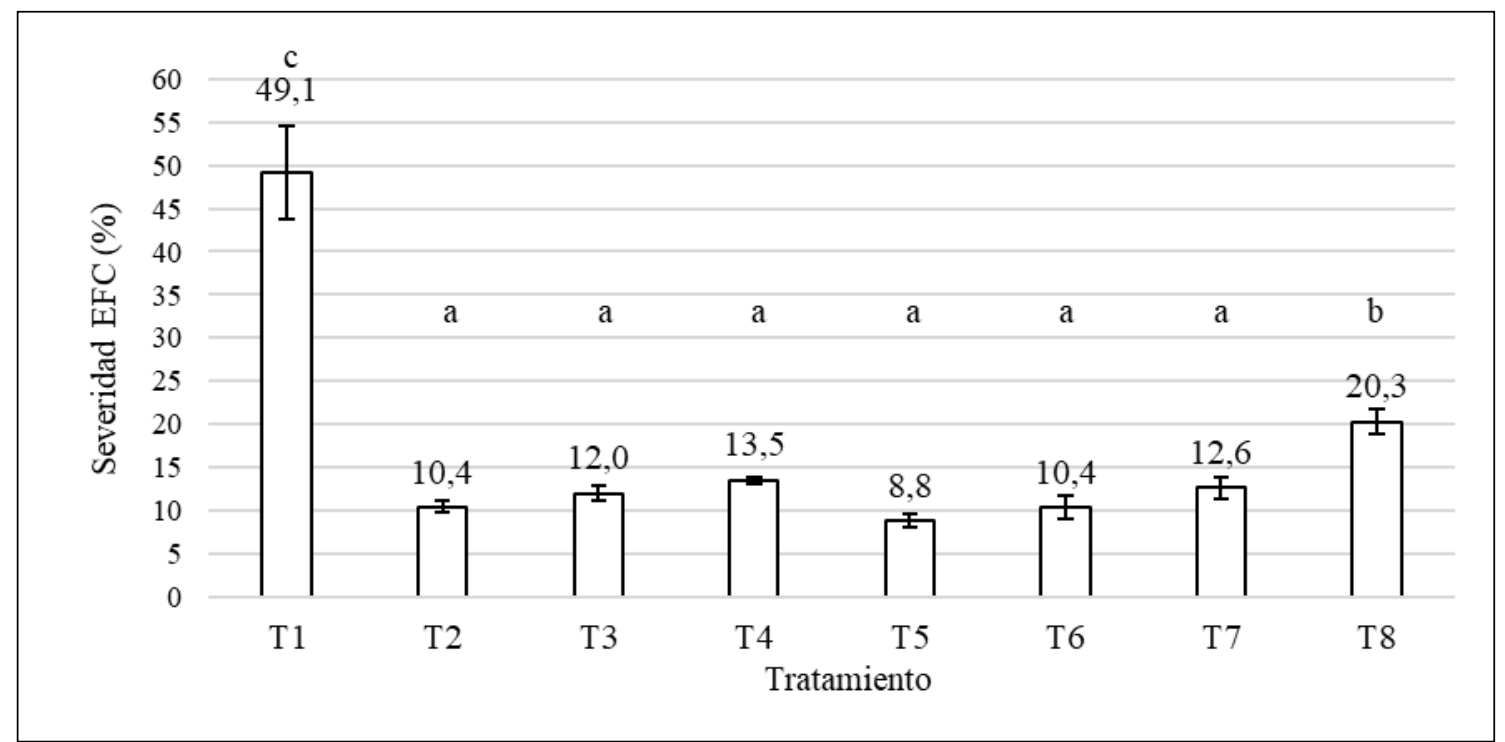

Figura 2. Porcentaje de severidad de enfermedades de final de ciclo en la soja posterior a la aplicación de los tratamientos. *Medias con una letra en común no son significativamente diferentes $(p>0.05)$; coeficiente de variación $=12,19 . \mathrm{T} 1=$ Testigo (agua); T2 $=$ Bixafen $12,5 \%+$ Protioconazole $17,5 \%+$ Trifloxistrobin $15 \%$ a $500 \mathrm{~mL} \mathrm{ha}^{-1}$; T3 = Picoxystrobin $10 \%$ + Protioconazole $11,67 \%$ a $600 \mathrm{~mL} \mathrm{ha}^{-1}$; T4 = Picoxystrobin $9 \%$ + Cyproconazole $4 \%$ a $600 \mathrm{~mL} \mathrm{ha}^{-1}$; T5 = Fluxapyroxad $5 \%$ + Epoxiconazole $5 \%$ + Pyraclostrobin $8,1 \%$ a $800 \mathrm{~mL} \mathrm{ha}^{-1}$; T6 = Benzovindiflupyr $15 \%$ + Azoxistrobina $30 \%$ a $200 \mathrm{~g}$ $\mathrm{ha}^{-1}+$ Difenoconazole $25 \%+$ Ciproconazole $15 \%$ a $250 \mathrm{~mL} \mathrm{ha}^{-1}$; T7 = Mancozeb $80 \%$ a $1500 \mathrm{~g} \mathrm{ha}^{-1}$; $\mathrm{T} 8=$ Carbendazim $50 \mathrm{FW}$ a $400 \mathrm{~mL} \mathrm{ha}^{-1}$.

Por otro lado, se encontraron diferencias significativas para la eficacia de control de los fungicidas (EC) $(\mathrm{p}<0,0001)$. Esta variable es indirectamente proporcional al valor de severidad, ya que se obtiene a partir de ella. Los tratamientos T5, T6 y T2 fueron los más eficaces para controlar las EFC con valores de 81,$8 ; 78,7$ y 78,6 \%, diferenciándose significativamente de los demás tratamientos. La menor EC se observó con el T8 con 58,5 \% (Figura 3).

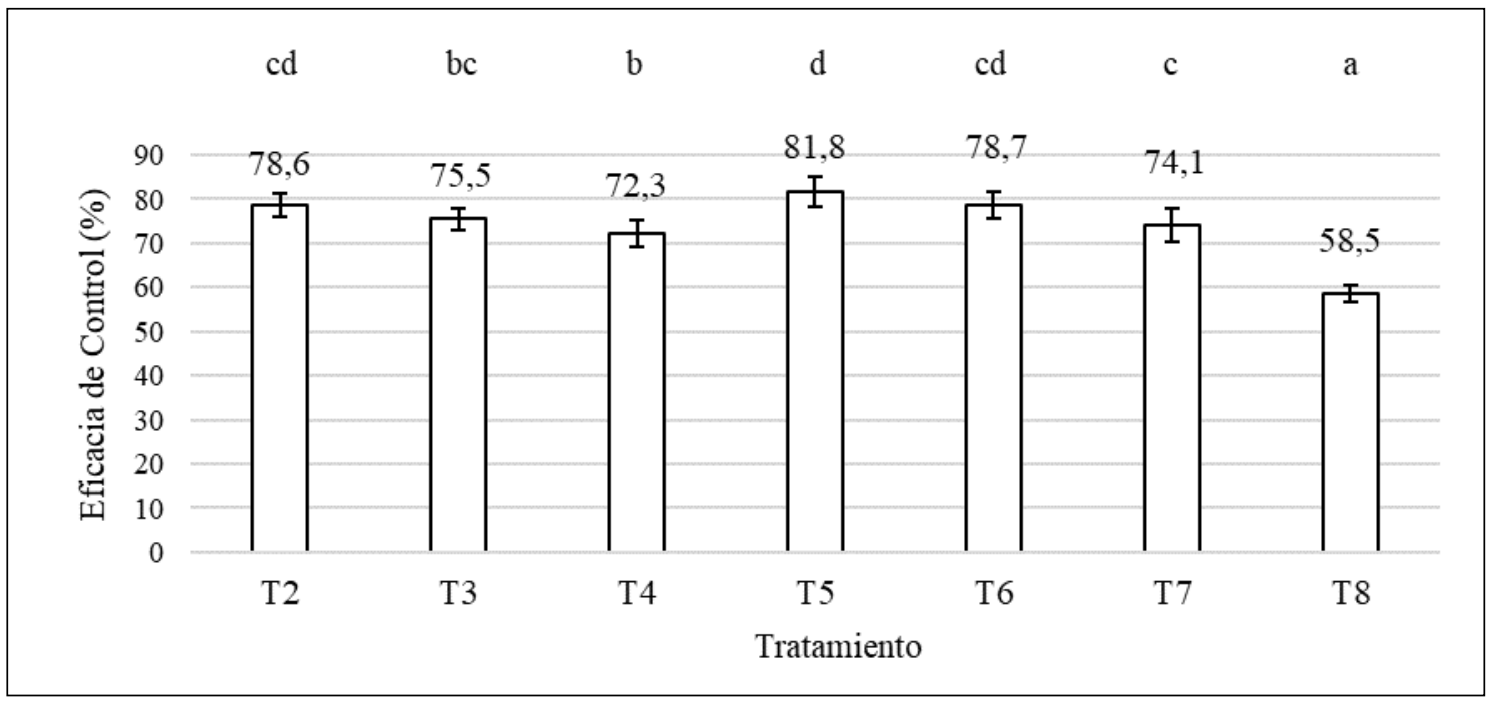

Figura 3. Porcentaje de eficacia de control de los fungicidas evaluados en soja. *Medias con una letra en común no son significativamente diferentes $(p>0,05)$; Coeficiente de variación $=10,2$. T1 $=$ Testigo (agua); T2 = Bixafen 12,5\% + Protioconazole 17,5\% + Trifloxistrobin $15 \%$ a $500 \mathrm{~mL} \mathrm{ha}^{-1}$; T3 = Picoxystrobin $10 \%$ + Protioconazole $11,67 \%$ a $600 \mathrm{~mL} \mathrm{ha}^{-1}$; T4 = Picoxystrobin $9 \%$ + Cyproconazole 4 $\%$ a $600 \mathrm{~mL} \mathrm{ha}^{-1}$; T5 = Fluxapyroxad $5 \%+$ Epoxiconazole $5 \%+$ Pyraclostrobin $8,1 \%$ a $800 \mathrm{~mL} \mathrm{ha}^{-1}$; T6 = Benzovindiflupyr $15 \%$ + Azoxistrobina $30 \%$ a $200 \mathrm{~g} \mathrm{ha}^{-1}+$ Difenoconazole $25 \%$ + Ciproconazole $15 \%$ a $250 \mathrm{~mL} \mathrm{ha}^{-1}$; T7 = Mancozeb $80 \%$ a $1500 \mathrm{~g} \mathrm{ha}^{-1}$; $\mathrm{T} 8=$ Carbendazim $50 \mathrm{FW}$ a $400 \mathrm{~mL} \mathrm{ha}^{-1}$. 


\section{Defoliación}

Se observaron diferencias significativas entre los tratamientos para la defoliación $(\mathrm{p}<0,0001)$, indicando que ésta fue afectada por la aplicación de tratamientos. Los tratamientos T5, T2, T6 y alcanzaron los menores valores de defoliación con 69,3, 72,4 y 72,5 \%, respectivamente, seguidamente por T7 y T4 con 76,6 y 78,2 \%. Por último, T1 (testigo) y T8, alcanzaron los mayores valores de defoliación (Figura 4).

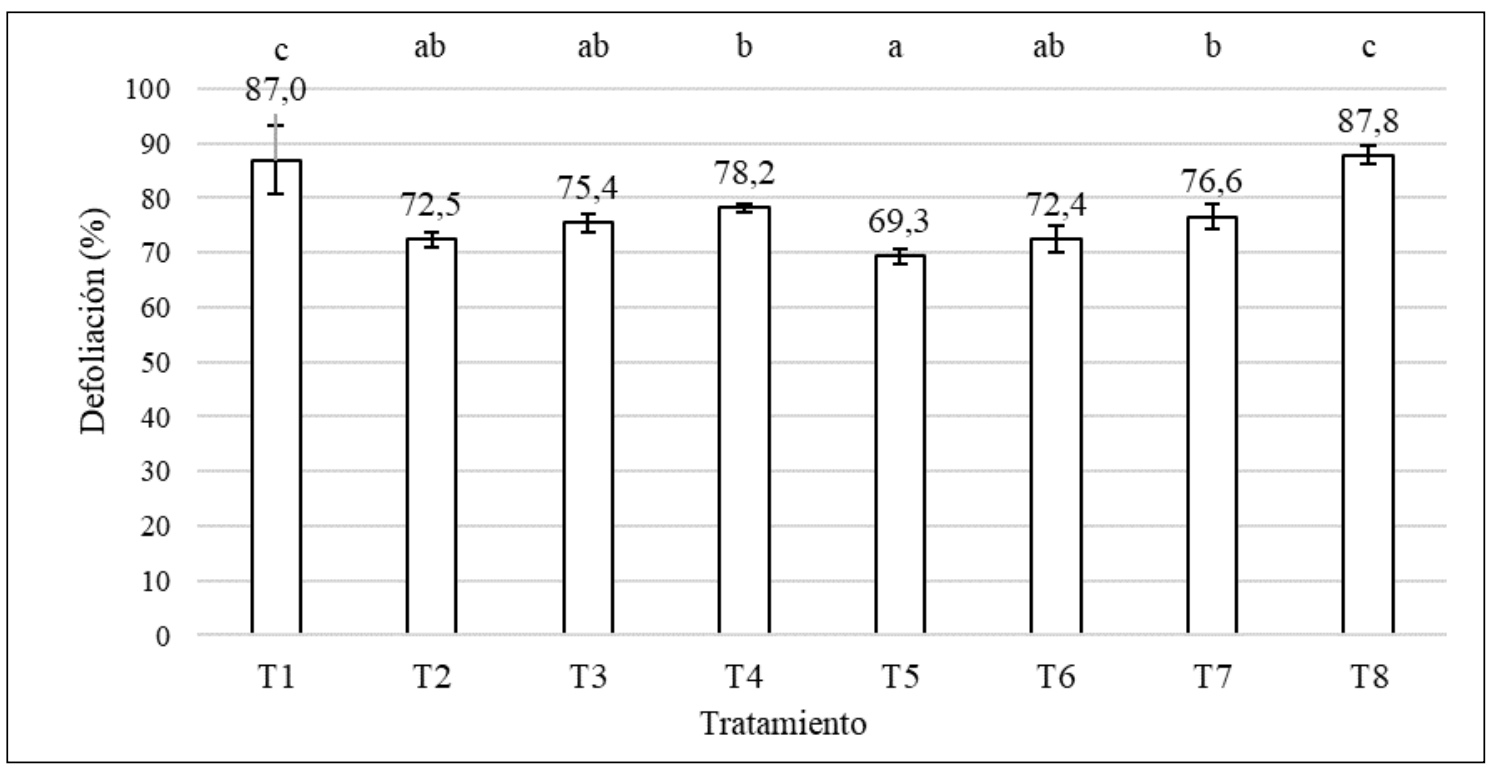

Figura 4. Porcentaje de defoliación de soja posterior a la aplicación de los tratamientos. *Medias con una letra en común no son significativamente diferentes $(p>0,05)$; Coeficiente de variación $=3,7$. T1 $=$ Testigo (agua); T2 = Bixafen 12,5\% + Protioconazole 17,5\% + Trifloxistrobin $15 \%$ a $500 \mathrm{~mL} \mathrm{ha}^{-1}$; T3 = Picoxystrobin $10 \%+$ Protioconazole $11,67 \%$ a $600 \mathrm{~mL} \mathrm{ha}^{-1}$; T4 = Picoxystrobin $9 \%+$ Cyproconazole $4 \%$ a $600 \mathrm{~mL} \mathrm{ha}^{-1}$; T5 = Fluxapyroxad $5 \%+$ Epoxiconazole $5 \%+$ Pyraclostrobin $8,1 \%$ a $800 \mathrm{~mL} \mathrm{ha}^{-1}$; T6 = Benzovindiflupyr $15 \%$ + Azoxistrobina $30 \%$ a $200 \mathrm{~g} \mathrm{ha}^{-1}+$ Difenoconazole $25 \%$ + Ciproconazole $15 \%$ a $250 \mathrm{~mL} \mathrm{ha}^{-1}$; T7 = Mancozeb $80 \%$ a $1500 \mathrm{~g} \mathrm{ha}^{-1}$; T8 = Carbendazim $50 \mathrm{FW} \mathrm{a} 400 \mathrm{~mL} \mathrm{ha}^{-1}$.

\section{Rendimiento y peso de mil semillas (PMS)}

Se observaron diferencias significativas entre los tratamientos para el rendimiento $(\mathrm{p}<0,0001)$ y el peso de mil semillas $(\mathrm{p}<0,0001)$. En la Tabla 2 se muestran los resultados de los rendimientos obtenidos, así como la diferencia de rendimiento con el testigo y el peso de mil semillas (PMS).

Los tratamientos T2, T3, T4, T5, T6 y T7 tuvieron el mismo efecto sobre el rendimiento, superando los $3200 \mathrm{~kg} \mathrm{ha}^{-1} \mathrm{y}$ diferencias con el testigo en más de $150 \mathrm{~kg} \mathrm{ha}^{-1}$. El T8 mostró el menor rendimiento, siendo estadísticamente igual al testigo, con una diferencia de $-7,9 \mathrm{~kg} \mathrm{ha}^{-1}$ (Tabla 2). Por otro lado, en cuanto al PMS, los fungicidas evaluados mostraron un efecto positivo sobre esta variable, excepto T8, el cual fue estadísticamente similar al testigo (Tabla 2).

El aumento del rendimiento observado con la aplicación de fungicidas se atribuye a una mayor sanidad de la lámina foliar en los periodos críticos del cultivo, que permitió a las plantas completar su desarrollo de manera más eficiente, con mayor captación de la radiación incidente (Zárate, 2017). Por su parte, Carmona (2010), señala qué las enfermedades del follaje en soja disminuyen significativamente el rendimiento entre un $11 \%$ a un $20 \%$, cuando las mismas no se controlaron con fungicidas foliares.

En este trabajo, si se toma como $100 \%$ al mayor rendimiento (obtenido con T5), que fue de $3318,8 \mathrm{Kg}^{-1} \mathrm{y}^{-1}$ se compara con los menores rendimientos, se puede observar que la pérdida de rendimiento al no aplicar fungicidas o al aplicar Carbendazim $50 \mathrm{FW}$ es de 7,9 a 9,1\%, lo cual está dentro del rango reportado por Carmona (2010). 
Tabla 2. Tratamiento, rendimiento, diferencia con el testigo y peso de mil semillas (PMS).

\begin{tabular}{cccccc}
\hline Tratamiento $^{\text {a }}$ & $\begin{array}{c}\text { Rendimiento } \\
\left(\mathrm{kg} \mathrm{ha}^{-1}\right)\end{array}$ & $\begin{array}{c}\text { Diferencia con el testigo } \\
\left(\mathrm{kg} \mathrm{ha}^{-1}\right)\end{array}$ & $\begin{array}{c}\text { PMS } \\
(\mathrm{g})\end{array}$ \\
\hline T1 & 3058,2 & $\mathrm{a}^{*}$ & - & 125,0 & $\mathrm{a}$ \\
T2 & 3288,7 & $\mathrm{~b}$ & 230,4 & 132,4 & $\mathrm{~b}$ \\
T3 & 3253,7 & $\mathrm{~b}$ & 195,5 & 131,2 & $\mathrm{~b}$ \\
T4 & 3217,2 & $\mathrm{~b}$ & 158,9 & 130,1 & $\mathrm{~b}$ \\
T5 & 3318,8 & $\mathrm{~b}$ & 260,6 & 133,4 & $\mathrm{~b}$ \\
T6 & 3288,7 & $\mathrm{~b}$ & 230,4 & 132,3 & $\mathrm{~b}$ \\
T7 & 3238,2 & $\mathrm{~b}$ & 179,9 & 130,7 & $\mathrm{~b}$ \\
T8 & 3050,3 & $\mathrm{a}$ & $-7,9$ & 124,7 & $\mathrm{a}$ \\
\hline Promedio & 3214,0 & - & - & 129,9 & - \\
C. V. $(\%)$ & 11,4 & - & - & 9,2 & - \\
\hline
\end{tabular}

*Medias con una letra en común no son significativamente diferentes $(\mathrm{p}>0,05)$.

a: T1 $=$ Testigo (agua); T2 = Bixafen 12,5\% + Protioconazole $17,5 \%+$ Trifloxistrobin $15 \%$ a $500 \mathrm{~mL}$ $\mathrm{ha}^{-1}$; T3 = Picoxystrobin $10 \%$ + Protioconazole $11,67 \%$ a $600 \mathrm{~mL} \mathrm{ha}^{-1} ; \mathrm{T} 4=$ Picoxystrobin $9 \%+\mathrm{Cy}-$ proconazole $4 \%$ a $600 \mathrm{~mL} \mathrm{ha}^{-1}$; T5 = Fluxapyroxad $5 \%$ + Epoxiconazole $5 \%+$ Pyraclostrobin $8,1 \%$ a $800 \mathrm{~mL} \mathrm{ha}^{-1}$; T6 = Benzovindiflupyr $15 \%+$ Azoxistrobina $30 \%$ a $200 \mathrm{~g} \mathrm{ha}^{-1}+$ Difenoconazole $25 \%+$ Ciproconazole $15 \%$ a $250 \mathrm{~mL} \mathrm{ha}^{-1} ; \mathrm{T} 7=$ Mancozeb $80 \%$ a $1500 \mathrm{~g} \mathrm{ha}^{-1} ; \mathrm{T} 8=$ Carbendazim $50 \mathrm{FW}$ a $400 \mathrm{~mL} \mathrm{ha}^{-1}$.

Las principales recomendaciones para el manejo de EFC son el uso de variedades resistentes y la aplicación de fungicidas a partir de la floración, sin embargo, como las EFC son causadas por un complejo de patógenos, la resistencia genética no es general para todas las enfermedades, por ejemplo, la mayoría de las variedades son susceptibles a la mancha marrón (Septoria glycines) y a la antracnosis (Colletotrichum spp.), y en algunos casos ya se han reportado variedades o líneas resistentes al tizón foliar y a la mancha púrpura de la semilla (Cercospora spp.) (Hartman et al., 2015). En Paraguay, se siembran variedades resistentes a la roya de la soja en el 1,47 \% del total del área de siembra (3 millones de hectáreas) (Ishikawa-Ishiwata y Furuya, 2021), mientras que, para las EFC, no existen líneas ni variedades resistentes reportadas (Enciso-Maldonado y FernándezGamarra, 2021). Por lo tanto, la aplicación de fungicidas seguirá siendo la principal herramienta del manejo de las EFC.

En este trabajo se realizaron las aplicaciones de fungicidas según se recomienda para el manejo de las EFC a nivel regional. En cuanto al uso de fungicidas en Paraguay, normalmente se realizan tres a cuatro aplicaciones por ciclo de cultivo en la primera época de siembra con foco principal para manejar a la roya de la soja, esto implica se traduce en un costo de entre 120 a 145 USD ha $^{-1}$ y entre 425 a 514 millones de dólares a nivel país (Enciso-Maldonado et al., 2021); Ishikawa-Ishiwata y Furuya (2021).

Otro punto importante es que, en Paraguay, las investigaciones de enfermedades de soja son limitadas, existen pocos o nulos estudios sobre la eficacia de control de fungicidas, sobre determinación de pérdidas y sobre umbrales económicos y de daño de las enfermedades de soja (Arrua et al., 2021, Enciso-Maldonado et al., 2021). Mientras que, las pérdidas ocasionadas por las EFC reportadas en Argentina se encuentran entre el 11 y $20 \%$ (Lavilla e Ivancovich, 2021). En este trabajo, al comparar la diferencia de rendimiento entre el mejor tratamiento (T5) con el testigo, existe una diferencia de 7,9\% $\left(260,6 \mathrm{~kg} \mathrm{ha}^{-1}\right)$. Considerando el precio promedio de la soja 270 USD ton ${ }^{-1}$ (marzo de 2020), esta pérdida representa unos 70,4 USD, lo cual hace que el control de la enfermedad sea más elevado que la perdida ocasionada.

A partir de este trabajo, se recomienda realizar ensayos para determinar pérdidas ocasionadas por las diferentes enfermedades del complejo de las EFC en varias localidades del Paraguay, con ello se podrá reevaluar la factibilidad de utilizar fungicidas para su manejo o incluir nuevas tácticas de manejo, como los sistemas de predicción para tomar la decisión de aplicar fungicidas, y con ello, reducir el uso de estos y generar un ahorro para el agricultor. 


\section{CONCLUSIONES}

Los fungicidas tuvieron el mismo efecto sobre la severidad de las EFC, excepto el Carbendazim $50 \mathrm{FW}$. Numéricamente se destacó el T5 (fluxapyroxad + epoxiconazole + pyraclostrobin).

La mayor eficacia de control de EFC se obtuvo con los fungicidas del T5 (fluxapyroad + epoxiconazole + pyraclostrobin), T6 (benzovindiflupyr + azoxistrobina, difenoconazole + ciproconazole \%) y T2 (bixafen + protioconazole + trifloxistrobin).

La menor defoliación del cultivo se obtuvo con T5 (Fluxapyroxad + epoxiconazole + pyraclostrobin), T2 (Bixafen + Protioconazole + Trifloxistrobin), T6 (benzovindiflupyr + azoxistrobina, difenoconazole + ciproconazole) y T3 (Picoxystrobin + Protioconazole).

Carbendazim no fue efectivo en el control de la enfermedad, obtuvo una alta defoliación y tuvo rendimientos y peso de mil semillas similares al testigo, a diferencia de los otros tratamientos que permiten un incremento del rendimiento y del peso de mil semillas.

\section{REFERENCIAS}

Arrua, A.A., Lopez-Nicora, H. D., Ríos, D. F., Grabowski, C. J., Cazal-Martínez, C. y Kohli, M. M. (2021). El impacto de las enfermedades fúngicas en el cultivo de soja (Glycine max) en Paraguay ¿es posible estimar las pérdidas?. Investigaciones y Estudios-UNA, 12 (1): 34-46.

Carmona, M.A. (2010). Enfermedades de fin de ciclo y mancha ojo de rana en el cultivo de soja: desarrollo de un sistema de puntuación y determinación del umbral de control (Doctoral dissertation, Universidad Nacional de La Plata).

Enciso-Maldonado, G.A y Fernández-Gamarra, M.A. (2021). Cercospora kikuchii ¿Patógeno potencial de la soja? Revista Impacto - FACYT UNI, 1 (1): 69-72.

Enciso-Maldonado, G.A, Maidana-Ojeda, M., Schlickmann-Tank, J.A., Montoya-García, C.O., Páez-Ranoni, H.J., Fernández-Riquelme, F. y Domínguez-Sanabria, J.A. (2019). Fungicidas sitio-específicos combinados con Mancozeb para el control de la roya asiática de la soya. Revista Mexicana de Fitopatología, Mexican Journal of Phytopathology, 37 (1).

Enciso-Maldonado, G.A., Sanabria-Velázquez, A.D., Schlickmann-Tank, J.A. y Maidana-Ojeda, M. (2021). Una carrera contra la perdida de la eficacia de fungicidas: 20 años de manejo de la roya asiática de la soja en Paraguay. Investigaciones y Estudios UNA, 12 (2): 59-61.

Hartman, G.L., Rupe, J.C., Sikora, E.J., Domier, L.L., Davis, J.A. y Steffey K.L. (Eds.). (2015). Compendium of soybean diseases and pests. St. Paul, MN: American Phytopathological Society. https://doi.org/10.1094/9780890544754

Hirano, M., Hikishima, M., Silva, A.J.D., Xavier, S.A. y Canteri, M.G. 2010. Validation of diagrammatic scale for estimating defoliation caused by the Asian rust in soybeans. Summa Phytopathologica, 36 (3): 248250.

Ishikawa-Ishiwata, Y., y Furuya, J. (2021). Fungicide Cost Reduction with Soybean Rust-Resistant Cultivars in Paraguay: A Supply and Demand Approach. Sustainability, 13 (2): 887p.

Ivancovich, A. y Botta, G. (2003). Enfermedades de la soja. Manejo Integrado de Enfermedades en Cultivos Extensivos. Jornadas Técnicas, pp 85-86.

Lavilla, M. e Ivancovich, A. (2021). Relación entre enfermedades y rendimiento de granos de soja. Agronomía Mesoamericana, 32 ( 2): 479-486 pp. https://doi.org/10.15517/am.v32i2.44057

Martins, M.C., Guerzoni, R.A., Câmara, G.M.D.S., Mattiazzi, P., Lourenço, S.A. y Amorim, L. (2004). Escala diagramática para a quantificação do complexo de doenças foliares de final de ciclo em soja. Fitopatologia Brasileira, 29 (2): 179-184. https://doi.org/10.1590/S0100-41582004000200009

Toledo, R. E. (2018). Ecofisiología, rendimiento y calidad de la soja. Facultad de Ciencias Agropecuarias, Universidad Nacional de Córdoba. Materiales Didácticos FCA.

Zárate, J. F. (2017). Evaluación de la aplicación de fungicidas en la severidad de las enfermedades de fin de ciclo en soja y su impacto en los rendimientos (Doctoral dissertation, Universidad Nacional de La Plata). 